\section{Moving in the Anthropocene: Global reductions in terrestrial mammalian movements}

\author{
Marlee A. Tucker, ${ }^{1,2 *}$ Katrin Böhning-Gaese, ${ }^{1,2}$ William F. Fagan, ${ }^{3,4}$ John M. Fryxell, ${ }^{5}$ \\ Bram Van Moorter, ${ }^{6}$ Susan C. Alberts, ${ }^{7}$ Abdullahi H. Ali, ${ }^{8}$ Andrew M. Allen,,${ }^{9,10}$ \\ Nina Attias, ${ }^{11}$ Tal Avgar, ${ }^{12}$ Hattie Bartlam-Brooks, ${ }^{13}$ Buuveibaatar Bayarbaatar, ${ }^{14}$ \\ Jerrold L. Belant, ${ }^{15}$ Alessandra Bertassoni, ${ }^{16}$ Dean Beyer, ${ }^{17}$ Laura Bidner, ${ }^{18}$ \\ Floris M. van Beest, ${ }^{19}$ Stephen Blake, ${ }^{20,21}$ Niels Blaum, ${ }^{22}$ Chloe Bracis, ${ }^{1,2}$ \\ Danielle Brown, ${ }^{23}$ P. J. Nico de Bruyn, ${ }^{24}$ Francesca Cagnacci, ${ }^{25,26}$
} Justin M. Calabrese, ${ }^{3,27}$ Constança Camilo-Alves, ${ }^{28,29}$ Simon Chamaillé-Jammes, ${ }^{30}$ Andre Chiaradia, ${ }^{31,32}$ Sarah C. Davidson, ${ }^{33,20}$ Todd Dennis, ${ }^{34}$ Stephen DeStefano, ${ }^{35}$ Duane Diefenbach, ${ }^{36}$ Iain Douglas-Hamilton, ${ }^{37,38}$ Julian Fennessy, ${ }^{39}$ Claudia Fichtel, ${ }^{40}$ Wolfgang Fiedler, ${ }^{20}$ Christina Fischer, ${ }^{41}$ Hya Fischhoff, ${ }^{42}$ Christen H. Fleming, ${ }^{3,27}$ Adam T. Ford, ${ }^{43}$ Susanne A. Fritz, ${ }^{1,2}$ Benedikt Gehr, ${ }^{44}$ Jacob R. Goheen, ${ }^{45}$ Eliezer Gurarie, ${ }^{3,46}$ Mark Hebblewhite, ${ }^{47}$ Marco Heurich, ${ }^{48,49}$ A. J. Mark Hewison,,${ }^{50}$ Christian Hof, ${ }^{1}$ Edward Hurme, ${ }^{3}$ Lynne A. Isbell, ${ }^{18,51}$ René Janssen, ${ }^{52}$ Florian Jeltsch, ${ }^{22}$ Petra Kaczensky, ${ }^{6,53}$ Adam Kane, ${ }^{54}$ Peter M. Kappeler, ${ }^{40}$ Matthew Kauffman, ${ }^{55}$ Roland Kays, ${ }^{56,57}$ Duncan Kimuyu, ${ }^{58}$ Flavia Koch, ${ }^{40,59}$ Bart Kranstauber, ${ }^{44}$ Scott LaPoint, ${ }^{20,60}$ Peter Leimgruber, ${ }^{27}$ John D. C. Linnell, ${ }^{6}$ Pascual López-López, ${ }^{61}$ A. Catherine Markham, ${ }^{62}$ Jenny Mattisson, ${ }^{6}$ Emilia Patricia Medici, ${ }^{63,64}$ Ugo Mellone, ${ }^{65}$ Evelyn Merrill, ${ }^{12}$ Guilherme de Miranda Mourão, ${ }^{66}$ Ronaldo G. Morato, ${ }^{67}$ Nicolas Morellet, ${ }^{50}$ Thomas A. Morrison, ${ }^{68}$ Samuel L. Díaz-Muñoz, ${ }^{69,70}$ Atle Mysterud, ${ }^{71}$ Dejid Nandintsetseg, ${ }^{1,2}$ Ran Nathan, ${ }^{72}$ Aidin Niamir, ${ }^{1}$ John Odden, ${ }^{73}$ Robert B. O'Hara, ${ }^{1,74}$ Luiz Gustavo R. Oliveira-Santos, ${ }^{75}$ Kirk A. Olson, ${ }^{14}$ Bruce D. Patterson, ${ }^{76}$ Rogerio Cunha de Paula, ${ }^{67}$ Luca Pedrotti, ${ }^{77}$ Björn Reineking, ${ }^{78,79}$ Martin Rimmler, ${ }^{80}$ Tracey L. Rogers, ${ }^{81}$ Christer Moe Rolandsen, ${ }^{6}$ Christopher S. Rosenberry, ${ }^{82}$ Daniel I. Rubenstein, ${ }^{83}$ Kamran Safi, ${ }^{20,84}$ Sonia Saïd, ${ }^{85}$ Nir Sapir, ${ }^{86}$ Hall Sawyer, ${ }^{87}$ Niels Martin Schmidt, ${ }^{19,88}$ Nuria Selva, ${ }^{89}$ Agnieszka Sergiel, ${ }^{89}$ Enkhtuvshin Shiilegdamba, ${ }^{14}$ João Paulo Silva, ${ }^{90,91,92}$ Navinder Singh, ${ }^{9}$ Erling J. Solberg, ${ }^{6}$ Orr Spiegel, ${ }^{93}$ Olav Strand, ${ }^{6}$ Siva Sundaresan, ${ }^{94}$ Wiebke Ullmann, ${ }^{22}$ Ulrich Voigt, ${ }^{95}$ Jake Wall, ${ }^{37}$ David Wattles, ${ }^{35}$ Martin Wikelski, ${ }^{20,84}$ Christopher C. Wilmers, ${ }^{96}$ John W. Wilson, ${ }^{97}$ George Wittemyer, ${ }^{37,98}$ Filip Zięba, ${ }^{99}$ Tomasz Zwijacz-Kozica, ${ }^{99}$ Thomas Mueller ${ }^{1,2,27 *}$

Animal movement is fundamental for ecosystem functioning and species survival, yet the effects of the anthropogenic footprint on animal movements have not been estimated across species. Using a unique GPS-tracking database of 803 individuals across 57 species, we found that movements of mammals in areas with a comparatively high human footprint were on average one-half to one-third the extent of their movements in areas with a low human footprint. We attribute this reduction to behavioral changes of individual animals and to the exclusion of species with long-range movements from areas with higher human impact. Global loss of vagility alters a key ecological trait of animals that affects not only population persistence but also ecosystem processes such as predator-prey interactions, nutrient cycling, and disease transmission.

W ith approximately 50 to $70 \%$ of Earth's land surface currently modified for human activities (I), patterns of biodiversity and ecosystem functions worldwide are changing (2). The expanding footprint of human activities not only is causing the loss of habitat and biodiversity, but is also affecting how animals move through fragmented and disturbed habitats. The extent to which animal movements are affected by anthropogenic effects on the structure and composition of landscapes and resource changes has been explored only in local geographic regions or within single species. Such studies typically report decreasing animal movements-for example, as a result of habitat fragmentation, barrier effects, or resource changes (3-6) -with only a few studies reporting longer migration routes $(7,8)$.

We conducted a global comparative study of how the human footprint affects movements of terrestrial nonvolant mammals, using Global Positioning System (GPS) location data of 803 individuals from 57 mammal species (Fig. 1 and movements as a result of habitat loss or altered table S2). Mean species mass ranged from 0.49 to $3940 \mathrm{~kg}$ and included herbivores, carnivores, and omnivores $(n=28,11$, and 18 species, respectively). For each individual, we annotated locations with the Human Footprint Index (HFI), an index with a global extent that combines multiple proxies of human influence: the extent of built environments, crop land, pasture land, human population density, nighttime lights, railways, roads, and navigable waterways (9) (see supplementary materials for details). The HFI ranges from 0 (natural environments: e.g., the Brazilian Pantanal) to 50 (high-density built environments: e.g., New York City).

In addition to the human footprint, we included other covariates that are known to influence mammalian movements. Because individuals may need to cover a larger area to gather sufficient resources, mammals generally move farther in environments with lower productivity (10). To capture this effect, we annotated locations with the Normalized Difference Vegetation Index (NDVI), a well-established, satellite-derived measure of resource abundance for both herbivores and carnivores (11). Because an allometric scaling relationship shows that animals of greater body size usually move farther (12), and because diet may influence movements as a result of differences in foraging costs and availability of resource types $(13,14)$, we annotated the database with species averages for body size and dietary guild (i.e., carnivore, herbivore, or omnivore).

We then calculated displacements as the distance between subsequent GPS locations of each individual at nine time scales (15) ranging from 1 hour to 10 days. For each individual at each time scale, we calculated the 0.5 and 0.95 quantile of displacement. The combination of different time scales and quantile allowed us to examine the effect of the human footprint on both the median ( 0.5 quantile) and long-distance ( 0.95 quantile) movements for within-day movements (e.g., 1-hour time scale) up to longer time displacements of more than 1 week (e.g., 10-day time scale). We used linear mixed-effects models that, in addition to all covariates (i.e., NDVI, body mass, diet), also accounted for taxonomy and spatial autocorrelation (see supplementary materials for details).

We found strong negative effects of the human footprint on median and long-distance displacements of terrestrial mammals (Fig. 2, Fig. 3A, and table S3). Displacements of individuals (across species) living in areas of high footprint (HFI = 36) were shorter than displacements of individuals living in areas of low footprint $(\mathrm{HFI}=0)$ by as much as a factor of 3 . For example, median displacements for carnivores over 10 days were $3.3 \pm$ $1.4 \mathrm{~km}(\mathrm{SE})$ in areas of high footprint versus $6.9 \pm 1.3 \mathrm{~km}$ in areas of low footprint (Fig. 2A and table S3). Likewise, the maximum displacement distances for carnivores at the 10-day scale averaged $6.6 \pm 1.4 \mathrm{~km}$ in areas of high footprint versus $21.5 \pm 1.4 \mathrm{~km}$ in areas of low footprint (Fig. 2B and table S3). The effect was significant on all temporal scales with 8 hours or more between locations. 
The effect was not significant at shorter time scales (Fig. 3A, 1 to 4 hours), which suggests that the human footprint affects ranging behavior and area use over longer time scales, rather than altering individual travel speeds (i.e., individuals may travel at the same speed if measured across short time intervals, but have more tortuous movements in areas of higher human footprint and thus remain in the same locale if displacement is measured across longer time intervals).

Reduction in movement may be attributable to (i) an individual-behavioral effect, where individuals alter their movements relative to the human footprint, or (ii) a species occurrence effect, where certain species that exhibit long-range movement simply do not occur in areas of high human footprint. To disentangle these two effects, we ran additional models where we separated the HFI into two components: (i) the individualbehavioral effect represented by the individual variability of HFI relative to the species mean (i.e., the individual HFI minus the species mean HFI), and (ii) the species occurrence effect as the mean HFI for each species. Results from the twocomponent model indicate behavioral as well as species effects. We found a significant behavioral effect on median displacements and on longdistance displacements (0.95 quantile) at most time scales (from 8 hours to 10 days) (fig. S2A and table S4). The species occurrence effect was significant only over longer time scales (128- and 256-hour periods, or 5 and 10 days, respectively) (fig. S2B and table S4). However, we note that the estimate of the species occurrence effect is conservative because our model incorporated taxonomy as a random effect. Some variability in the data may have been accounted for by the specieslevel random effect rather than the species-level HFI (see table S3).

In addition to the human footprint effect, body mass, dietary guild, and resource availability were also related to movement distances. First, as expected from allometric scaling and established relationships of body size with home range size (14) and migration distance (16), larger species traveled farther than smaller species (Fig. 3C and tables S3 and S4). Second, we found a negative

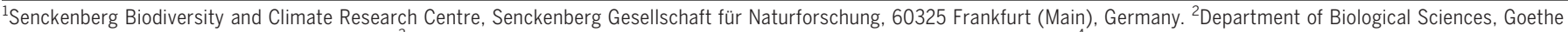

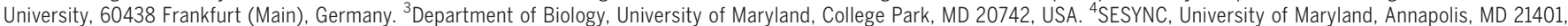
USA. ${ }^{5}$ Department of Integrative Biology, University of Guelph, Guelph, Ontario N1G 2W1, Canada. ${ }^{6}$ Norwegian Institute for Nature Research, P.0. Box 5685 Torgard, N0-7485

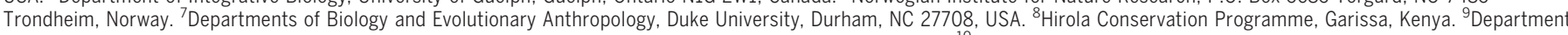
of Wildlife, Fish and Environmental Studies, Swedish University of Agricultural Sciences, Umeå 90183, Sweden. ${ }^{10}$ Institute for Water and Wetland Research, Department of Animal

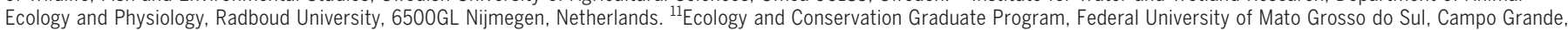

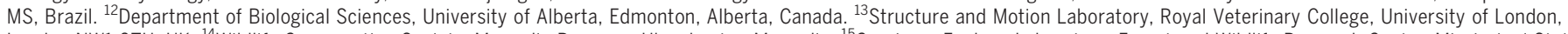

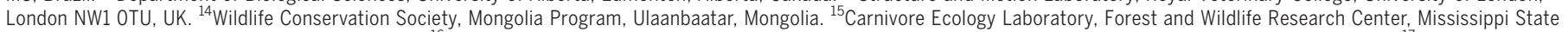
University, Box 9690, Mississippi State, MS, USA. ${ }^{16}$ Animal Biology Postgraduate Program, São Paulo State University, São José do Rio Preto, SP 15054-000, Brazil. ${ }^{17}$ Michigan Department of Natural Resources, 1990 U.S. 41 South, Marquette, MI 49855, USA. ${ }^{18}$ Department of Anthropology, University of California, Davis, CA 95616, USA. ${ }^{19}$ Department of

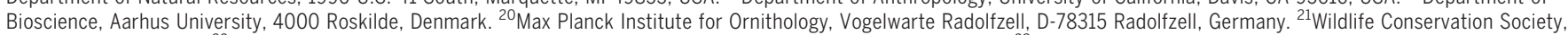
Bronx, NY 10460, USA. ${ }^{22}$ University of Potsdam, Plant Ecology and Nature Conservation, 14476 Potsdam, Germany. ${ }^{23}$ Department of Biology, Middle Tennessee State University,

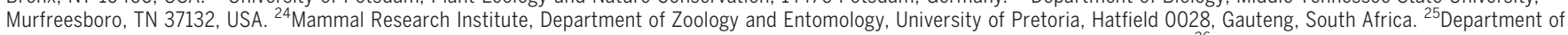

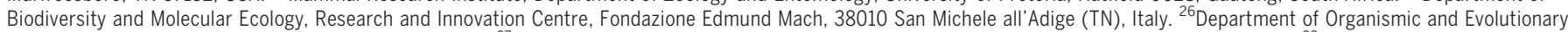

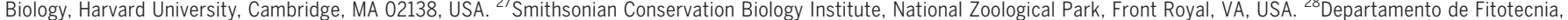
Universidade de Évora, Pólo da Mitra, 7002-554 Évora, Portugal. ${ }^{29}$ ICAAM-Institute of Mediterranean Agricultural and Environmental Sciences, University of Évora, Évora, Portugal.

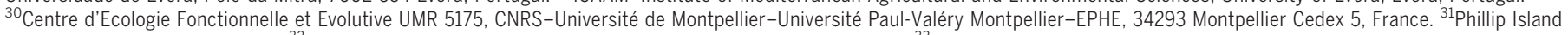

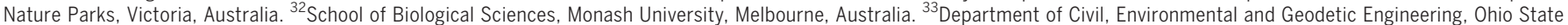
University, Columbus, OH 43210, USA. ${ }^{34}$ Department of Biology, Fiji National University, P.O. Box 5529, Natabua, Lautoka, Fiji Islands. ${ }^{35}$ U.S. Geological Survey, Massachusetts

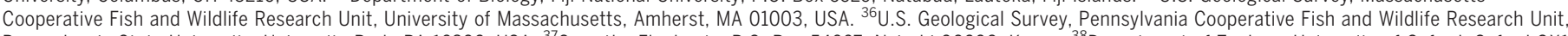

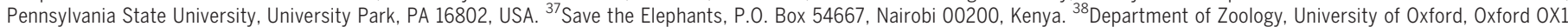
3PS, UK. ${ }^{39}$ Giraffe Conservation Foundation, P.0. Box 86099, Eros, Namibia. ${ }^{40}$ German Primate Center, Behavioral Ecology and Sociobiology Unit, 37077 Göttingen, Germany. ${ }^{41}$ Restoration Ecology, Department of Ecology and Ecosystem Management, Technische Universität München, 85354 Freising, Germany. ${ }^{42}$ Cary Institute of Ecosystem Studies, Millbrook, NY 12545, USA. ${ }^{43}$ Irving K. Barber School of Arts and Sciences, Unit 2: Biology, University of British Columbia, Okanagan Campus, Kelowna, BC V1V 1V7, Canada. ${ }^{44}$ Department of Evolutionary Biology and Environmental Studies, University of Zurich, 8057 Zurich, Switzerland. ${ }^{45}$ Department of Zoology and Physiology, University of Wyoming,

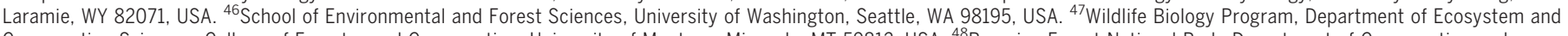
Conservation Sciences, College of Forestry and Conservation, University of Montana, Missoula, MT 59812, USA. ${ }^{48}$ Bavarian Forest National Park, Department of Conservation and

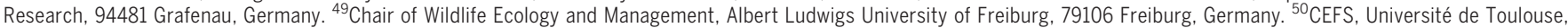
INRA, Castanet Tolosan, France. ${ }^{51}$ Animal Behavior Graduate Group, University of California, Davis, CA 95616, USA. ${ }^{52}$ Bionet Natuuronderzoek, 6171EL Stein, Netherlands.

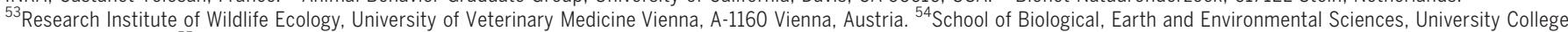

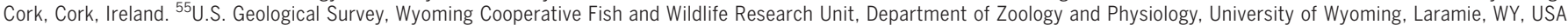

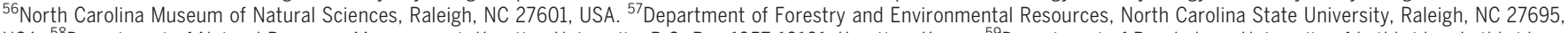

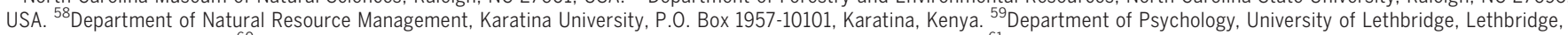
Alberta T1K 3M4, Canada. ${ }^{60}$ Lamont-Doherty Earth Observatory, Columbia University, Palisades, NY 10964, USA. ${ }^{61}$ Cavanilles Institute of Biodiversity and Evolutionary Biology, Terrestrial Vertebrates Group, University of Valencia, E-46980 Paterna, Valencia, Spain. ${ }^{62}$ Department of Anthropology, Stony Brook University, Stony Brook, NY 11794, USA.

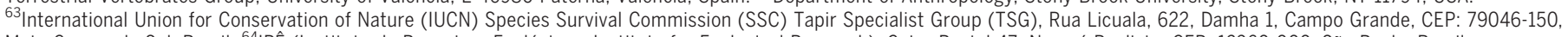
Mato Grosso do Sul, Brazil. ${ }^{64}$ IPÊ (Instituto de Pesquisas Ecológicas; Institute for Ecological Research), Caixa Postal 47, Nazaré Paulista, CEP: 12960-000, São Paulo, Brazil. ${ }^{65}$ Vertebrates Zoology Research Group, Departamento de Ciencias Ambientales y Recursos Naturales, University of Alicante, Alicante, Spain. ${ }^{66}$ Embrapa Pantanal, Corumbá,

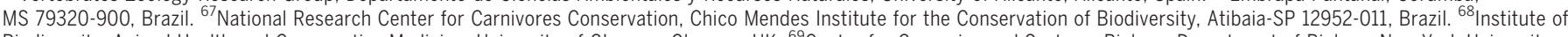

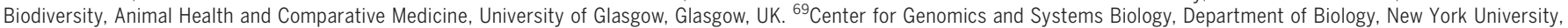

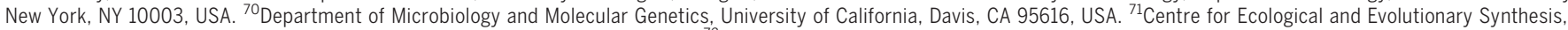

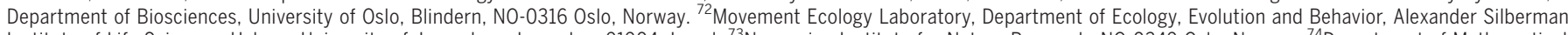

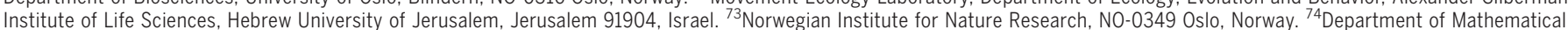

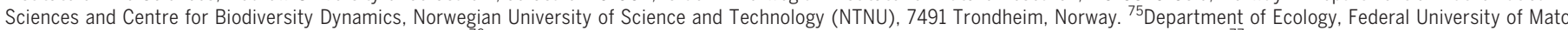
Grosso do Sul, Campo Grande, MS 79070-900, Brazil. ${ }^{76}$ Integrative Research Center, Field Museum of Natural History, Chicago, IL 60605, USA. ${ }^{77}$ Consorzio Parco Nazionale dello

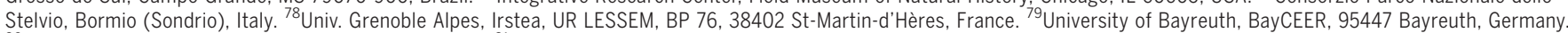

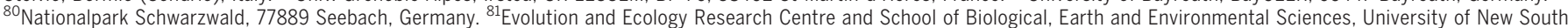

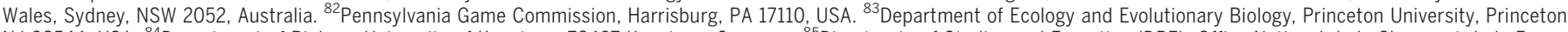

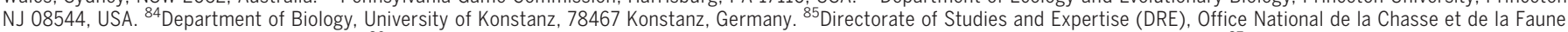

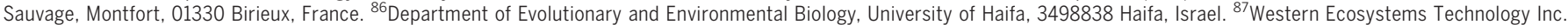
Laramie, WY 82070, USA. ${ }^{88}$ Arctic Research Centre, Aarhus University, 8000 Aarhus C, Denmark. ${ }^{89}$ Institute of Nature Conservation Polish Academy of Sciences, 31-120 Krakow,

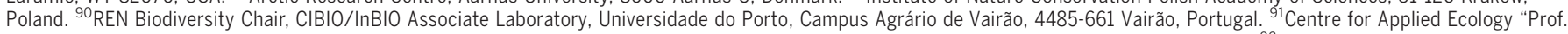

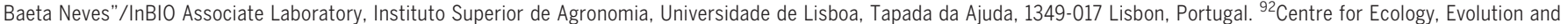

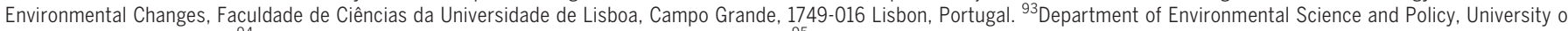

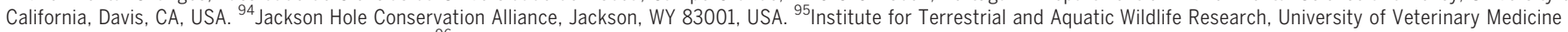

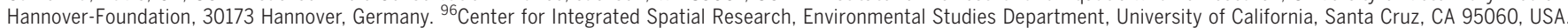

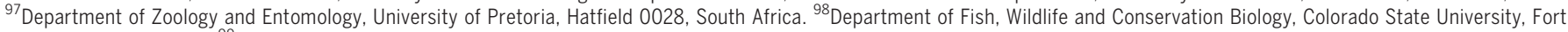
Collins, C0 80523, USA. ${ }^{99}$ Tatra National Park, 34-500 Zakopane, Poland.

*Corresponding author. Email: tucker.marlee@gmail.com (M.A.T.); thomas.mueller@senckenberg.de (T.M.) 


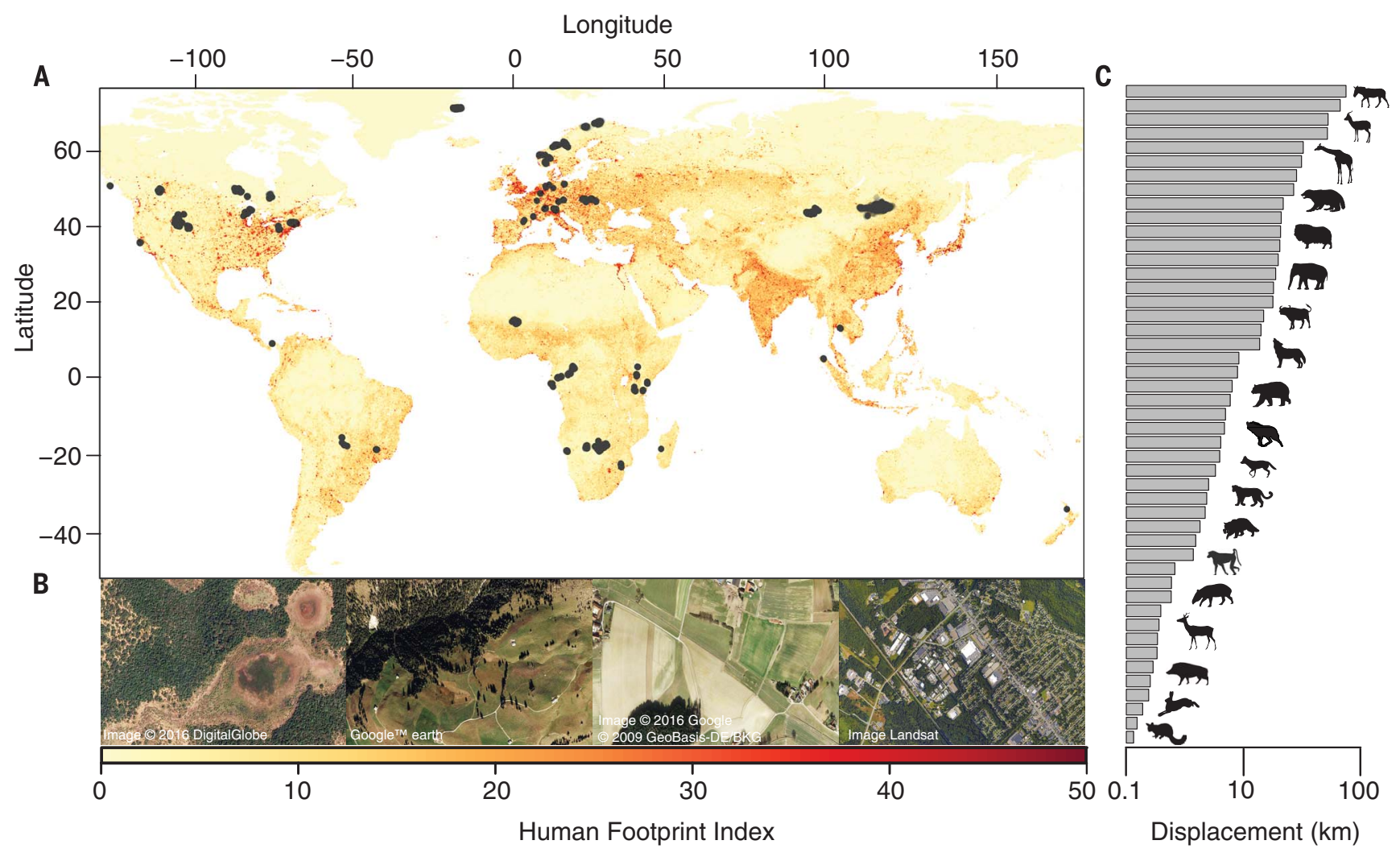

Fig. 1. Locations from the GPS tracking database and the Human Footprint Index. (A) GPS relocations of 803 individuals across 57 species plotted on the global map of the Human Footprint Index (HFI) spanning from 0 (low; yellow) to 50 (high; red). (B) Examples of landscapes under $\mathrm{HFI}=2$ (the Pantanal, Brazil), HFI = 20 (Bernese Alps, Switzerland), $\mathrm{HFI}=30$ (Freising, Germany), and HFI = 42 (Albany, New York). (C) Species averages of 10-day long-distance displacement (0.95 quantile of individual displacements). Species (from top to bottom): Mongolian wild ass (Equus hemionus hemionus), Mongolian gazelle (Procapra gutturosa), giraffe (Giraffa camelopardalis), wolverine (Gulo gulo), muskox (Ovibos moschatus), African forest elephant (Loxodonta africana cyclotis), African buffalo (Syncerus caffer), wolf (Canis lupus), brown bear (Ursus arctos), maned wolf (Chrysocyon brachyurus), coyote (Canis latrans), leopard (Panthera pardus), wildcat (Felis silvestris), yellow baboon (Papio cynocephalus), tapir (Tapirus terrestris), roe deer (Capreolus capreolus), wild boar (Sus scrofa), European hare (Lepus europaeus), brushtail possum (Trichosurus vulpecula).
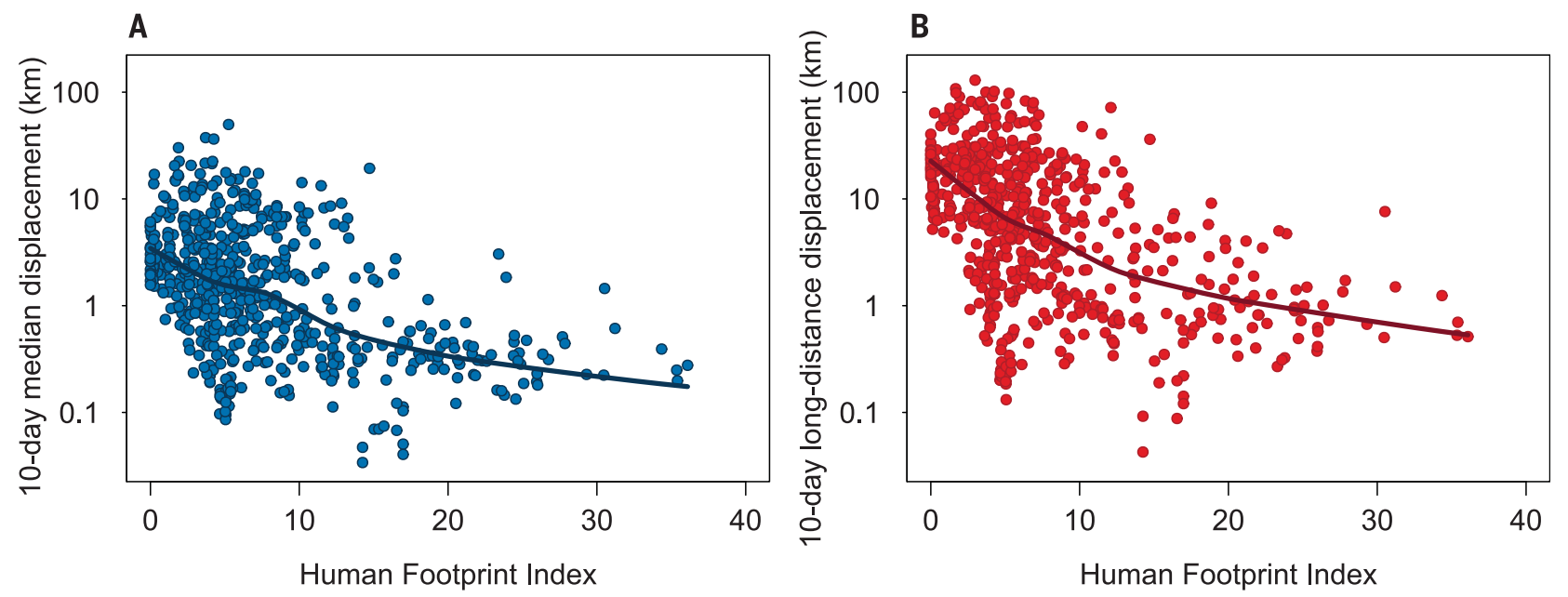

Fig. 2. Mammalian displacement in relation to the Human Footprint Index. (A) Median displacements; (B) long-distance (0.95 quantile) displacements. Both displacements decline with increasing HFI at the 10-day scale ( $n=48$ species and 624 individuals). Plots include a smoothing line from a locally weighted polynomial regression. An HFI value of 0 indicates areas of low human footprint; a value of 40 represents areas of high human footprint. 
A

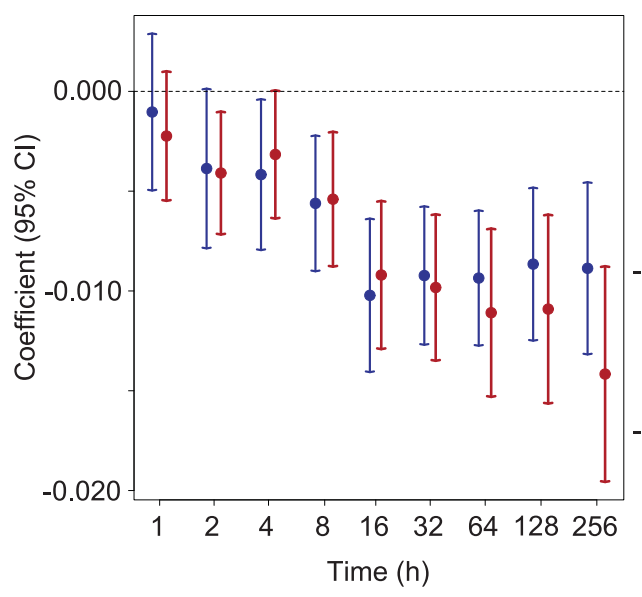

B

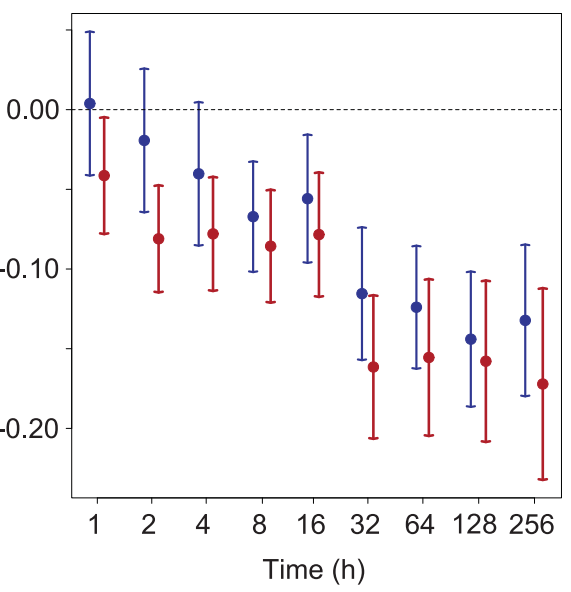

C

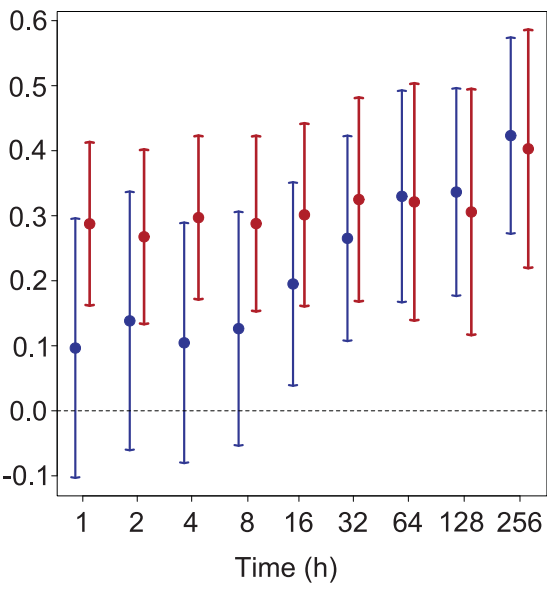

Fig. 3. Model coefficients (with confidence intervals) of linear mixed-effects models predicting mammalian displacements.

Coefficient values are shown for (A) Human Footprint Index (HFI),

(B) Normalized Difference Vegetation Index (NDVI), and (C) body mass.
Models were run for the median (blue) and long-distance (0.95 quantile; red) displacements of each individual calculated across different time scales. Where the error bars cross the horizontal line, the effect is not significant. See table S3 for details. relationship between resource availability and displacement distance, such that movements were on average shorter in environments with higher resources (Fig. 3B and tables S3 and S4). These results are consistent with reports of larger home range size (17) and longer migration distance (18) in mammals living in resource-poor environments. Finally, our analyses showed that carnivores traveled on average farther per unit time than herbivores and omnivores (tables S3 and S4). These results concur with prior understanding that carnivores have larger home range sizes (14) because they need to find mobile prey and compensate for energy conversion loss through the food web. For all of these variables, effects were significant across time scales longer than 8 hours for both median and long-distance displacements.

The reduction of mammalian movements in areas of high HFI likely stems from two nonexclusive mechanisms: (i) movement barriers such as habitat change and fragmentation $(19,20)$ and (ii) reduced movement requirements attributable to enhanced resources [e.g., crops, supplemental feeding, and water sources $(5,21)]$. Studies have shown both mechanisms at work with varying responses across populations or species (see table S5 for examples). In some cases, they act together on single individuals or populations. For example, red deer in Slovenia have smaller home ranges because of the enhancement of resources via supplemental feeding and the disturbance and fragmentation caused by the presence of roads (22).

Although these mechanisms can have differential effects on population densities [i.e., increases under supplementation (23) and decreases under fragmentation (24)], the consequences of reduced vagility affect ecosystems regardless of the underlying mechanisms and go far beyond the focal individuals themselves. Animal move- ments are essential for ecosystem functioning because they act as mobile links (25) and mediate key processes such as seed dispersal, food web dynamics (including herbivory and predator-prey interactions), and metapopulation and disease dynamics (26). Single-species or single-site studies have shown the severe effects of reduced vagility on these processes $(27,28)$. The global nature of reduced vagility across mammalian species that we demonstrate here suggests consequences for ecosystem functioning worldwide. Future landscape management should strive to maintain landscape permeability by including animal movement as a key conservation metric. Ultimately, because of the critical role of animal movement in human/ wildlife coexistence (29) and disease spread (30), the effects of reduced vagility may go beyond ecosystem functioning to directly affect human well-being.

\section{REFERENCES AND NOTES}

1. A. D. Barnosky et al., Nature 486, 52-58 (2012).

2. J. A. Foley et al., Science 309, 570-574 (2005).

3. H. Sawyer et al., J. Appl. Ecol. 50, 68-78 (2013).

4. S. Saïd, S. Servanty, Landsc. Ecol. 20, 1003-1012 (2005).

5. S. Prange, S. D. Gehrt, E. P. Wiggers, J. Mammal. 85, 483-490 (2004)

6. B. Jedrzejewska, H. Okarma, W. Jedrzejewski, L. Milkowski, J. Appl. Ecol. 31, 664-676 (1994).

7. L. A. Tigas, D. H. Van Vuren, R. M. Sauvajot, Biol. Conserv. 108 , 299-306 (2002).

8. J. Lenz et al., Proc. R. Soc. London Ser. B 278, 2257-2264 (2011)

9. O. Venter et al., Nat. Commun. 7, 12558 (2016)

10. T. Mueller et al., Glob. Ecol. Biogeogr. 20, 683-694 (2011)

11. N. Pettorelli et al., Clim. Res. 46, 15-27 (2011).

12. W. Jetz, C. Carbone, J. Fulford, J. H. Brown, Science 306 , 266-268 (2004)

13. B. K. McNab, Ecol. Monogr. 56, 1-19 (1986)

14. M. A. Tucker, T. J. Ord, T. L. Rogers, Glob. Ecol. Biogeogr. 23, 1105-1114 (2014)
15. J. M. Rowcliffe, C. Carbone, R. Kays, B. Kranstauber P. A. Jansen, Methods Ecol. Evol. 3, 653-662 (2012) 16. A. M. Hein, C. Hou, J. F. Gillooly, Ecol. Lett. 15, 104-110 (2012)

17. N. Morellet et al., J. Anim. Ecol. 82, 1326-1339 (2013).

18. C. S. Teitelbaum et al., Ecol. Lett. 18, 545-552 (2015).

19. J. F. Kamler et al., J. Mammal. 84, 989-995 (2003).

20. L. Fahrig, Funct. Ecol. 21, 1003-1015 (2007).

21. J. D. Jones et al., Ecol. Appl. 24, 1769-1779 (2014).

22. K. Jerina, J. Mammal. 93, 1139-1148 (2012).

23. J. S. Gilchrist, E. Otali, Can. J. Zool. 80, 1795-1802 (2002).

24. A. Benítez-López, R. Alkemade, P. A. Verweij, Biol. Conserv. 143, 1307-1316 (2010)

25. J. Lundberg, F. Moberg, Ecosystems 6, 87-98 (2003).

26. S. Bauer, B. J. Hoye, Science 344, 1242552 (2014)

27. I. Hanski, O. Ovaskainen, Nature 404, 755-758 (2000).

28. B. F. Allan, F. Keesing, R. S. Ostfeld, Conserv. Biol. 17, 267-272 (2003).

29. M. D. Graham, I. Douglas-Hamilton, W. M. Adams, P. C. Lee, Anim. Conserv. 12, 445-455 (2009)

30. J. M. Hassell, M. Begon, M. J. Ward, E. M. Fèvre, Trends Ecol. Evol. 32, 55-67 (2017)

\section{ACKNOWLEDGMENTS}

Supported by the Robert Bosch Foundation and additional funding sources (see supplementary text). The data reported in this paper are available at datadryad.org (doi: 10.5061/dryad. st350). M.A.T., T.M., K.B.-G., W.F.F., J.M.F., and B.V.M. conceived the manuscript; M.A.T. and T.M. conducted the analyses and wrote the first manuscript draft. Co-authors contributed data sets and assisted with writing the final version of the manuscript.

\section{SUPPLEMENTARY MATERIALS}

www.sciencemag.org/content/359/6374/466/suppl/DC1

Materials and Methods

Supplementary Text

Figs. S1 and S2

Tables S1 to S5

References (31-89)

15 February 2017; accepted 11 December 2017

10.1126/science.aam9712 


\section{Science}

\section{Moving in the Anthropocene: Global reductions in terrestrial mammalian movements}

Marlee A. Tucker, Katrin Böhning-Gaese, William F. Fagan, John M. Fryxell, Bram Van Moorter, Susan C. Alberts, Abdullahi $\mathrm{H}$. Ali, Andrew M. Allen, Nina Attias, Tal Avgar, Hattie Bartlam-Brooks, Buuveibaatar Bayarbaatar, Jerrold L. Belant, Alessandra Bertassoni, Dean Beyer, Laura Bidner, Floris M. van Beest, Stephen Blake, Niels Blaum, Chloe Bracis, Danielle Brown, P. J. Nico de Bruyn, Francesca Cagnacci, Justin M. Calabrese, Constança Camilo-Alves, Simon Chamaillé-Jammes, Andre Chiaradia, Sarah C. Davidson, Todd Dennis, Stephen DeStefano, Duane Diefenbach, lain Douglas-Hamilton, Julian Fennessy, Claudia Fichtel, Wolfgang Fiedler, Christina Fischer, llya Fischhoff, Christen H. Fleming, Adam T. Ford, Susanne A. Fritz, Benedikt Gehr, Jacob R. Goheen, Eliezer Gurarie, Mark Hebblewhite, Marco Heurich, A. J. Mark Hewison, Christian Hof, Edward Hurme, Lynne A. Isbell, René Janssen, Florian Jeltsch, Petra Kaczensky, Adam Kane, Peter M. Kappeler, Matthew Kauffman, Roland Kays, Duncan Kimuyu, Flavia Koch, Bart Kranstauber, Scott LaPoint, Peter Leimgruber, John D. C. Linnell, Pascual López-López, A. Catherine Markham, Jenny Mattisson, Emilia Patricia Medici, Ugo Mellone, Evelyn Merrill, Guilherme de Miranda Mourão, Ronaldo G. Morato, Nicolas Morellet, Thomas A. Morrison, Samuel L. Díaz-Muñoz, Atle Mysterud, Dejid Nandintsetseg, Ran Nathan, Aidin Niamir, John Odden, Robert B. O'Hara, Luiz Gustavo R. Oliveira-Santos, Kirk A. Olson, Bruce D. Patterson, Rogerio Cunha de Paula, Luca Pedrotti, Björn Reineking, Martin Rimmler, Tracey L. Rogers, Christer Moe Rolandsen, Christopher S. Rosenberry, Daniel I. Rubenstein, Kamran Safi, Sonia Saïd, Nir Sapir, Hall Sawyer, Niels Martin Schmidt, Nuria Selva, Agnieszka Sergiel, Enkhtuvshin Shiilegdamba, João Paulo Silva, Navinder Singh, Erling J. Solberg, Orr Spiegel, Olav Strand, Siva Sundaresan, Wiebke Ullmann, Ulrich Voigt, Jake Wall, David Wattles, Martin Wikelski, Christopher C. Wilmers, John W. Wilson, George Wittemyer, Filip Zieba, Tomasz Zwijacz-Kozica and Thomas Mueller

Science 359 (6374), 466-469.

DOI: $10.1126 /$ science.aam9712

\section{Restrictions on roaming}

Until the past century or so, the movement of wild animals was relatively unrestricted, and their travels contributed substantially to ecological processes. As humans have increasingly altered natural habitats, natural animal movements have been restricted. Tucker et al. examined GPS locations for more than 50 species. In general, animal movements were shorter in areas with high human impact, likely owing to changed behaviors and physical limitations. Besides affecting the species themselves, such changes could have wider effects by limiting the movement of nutrients and altering ecological interactions.

Science, this issue p. 466

ARTICLE TOOLS

SUPPLEMENTARY MATERIALS

REFERENCES http://science.sciencemag.org/content/359/6374/466

http://science.sciencemag.org/content/suppl/2018/01/24/359.6374.466.DC1

Use of this article is subject to the Terms of Service

Science (print ISSN 0036-8075; online ISSN 1095-9203) is published by the American Association for the Advancement of Science, 1200 New York Avenue NW, Washington, DC 20005. 2017 (C) The Authors, some rights reserved; exclusive licensee American Association for the Advancement of Science. No claim to original U.S. Government Works. The title Science is a registered trademark of AAAS. 
Use of this article is subject to the Terms of Service

Science (print ISSN 0036-8075; online ISSN 1095-9203) is published by the American Association for the Advancement of Science, 1200 New York Avenue NW, Washington, DC 20005. 2017 (C) The Authors, some rights reserved; exclusive licensee American Association for the Advancement of Science. No claim to original U.S. Government Works. The title Science is a registered trademark of AAAS. 